УДК 514.8

\title{
Необратимые деформации вращающегося цилиндра
}

C.B. $Ф$ ирсов

Институт машиноведения и металлургии ДВО РАН (г. Комсомольск-на-Амуре, Россия)

\section{Irreversible Deformations of a Rotating Cylinder}

\author{
S.V. Firsov
}

Institute of Engineering Science and Metallurgy, Far Eastern Branch of the Russian Academy of Sciences (Komsomolsk-on-Amur, Russia)

Рассматривается задача разгона среды, заполняющей цилиндр при учете необратимых деформаций ползучести и пластичности. Для сравнения рассмотрена задача вращения цилиндра без учета деформаций ползучести. Задача упругости решается аналитически, при начале вязкопластического течения, а также для ползучести производится численный расчет. Для моделирования процесса ползучести используется степенной закон Нортона с потенциалом по типу Мизеса. Для пластичности используется модель вязко-пластичности с потенциалом напряжений по типу Мизеса. При появлении пластического течения используется предположение о совместном протекании процессов накопления необратимых деформаций пластичности и ползучести. Делаются выводы относительно влияния деформаций ползучести на итоговое распределение напряжений в среде. Для цилиндрических сред с жестко зафиксированной левой границей наблюдается значительное снижение интенсивности напряжений. Для цилиндрической среды с полостью - менее значительное. Для свободной среды наблюдается перераспределение интенсивности напряжений внутри среды без их снижения.

Ключевые слова: малые деформации, вращающийся цилиндр, вязкоупругопластическое деформирование, закон ползучести Нортона.

\section{DOI 10.14258/izvasu(2018)4-21}

\section{Введение}

Вращающиеся цилиндры широко применяются в различных сферах. Исследование механических процессов, происходящих внутри них, представляет значительный интерес. Наиболее простым случаем является деформирование линейно-упругой среды. Первое решение задачи упругопластической деформации цилиндра получил в 1928 г. А. Надаи [1]. Подробно эта задача рассматривалась в работах $[2,3]$. В монографии Ю.Н. Работнова [4] приведено решение о вращающемся цилиндре в условиях ползучести. В работах [5-7] исследовалась ползучесть ортотропных цилиндров.
The problem of acceleration of a cylindrical medium with consideration of irreversible deformation of creep and plasticity is investigated. For comparison, the problem of a rotating cylinder without creep deformation is considered. The problem of elastic deformation is solved analytically for the case of viscoplastic deformation and numerically for the case of creep deformation. The Norton power low with continuous Mises type potential is used for modeling a process of creep deformation. The viscoplasticity model with Mises type stress potential is used for plastic deformation. When plastic flow occurs, it is assumed that the processes of accumulation of irreversible deformations of plasticity and creep take place together. The conclusions are made about the influence of creep deformation on the final distribution of stress. The significant decrease of stress intensity is observed for cylindrical mediums with rigid inclusion. However, the decrease is less significant for the hollow cylindrical medium. Also, the redistribution of stress intensity without decreasing is observed for the cylindrical medium with two free boundaries.

Key words: small deformation, rotating cylinder, viscoelastoplastic deforming, Norton power low.

\footnotetext{
^Работа выполнена в рамках государственного задания № 007-00285-18-00.
} 


\section{Построение математической модели}

Полагаем, что деформируемая среда заполняет бесконечный цилиндрический слой $R_{1} \leq r \leq R_{2}$. Область $r \leq R_{1}$ может быть жесткой (недеформируемой) либо полой. Граница $r=R_{2}$ может быть жестко зафиксированной либо свободной. Рассмотрим деформирование материала цилиндра за счет его вращения с угловой скоростью $\omega(t)$. Считаем, что полные деформации $d_{i j}$ в среде складываются из обратимых $e_{i j}$ и необратимых $p_{i j}$ деформаций. В цилиндрической системе координат $r, \varphi, z$ получим

$$
\begin{aligned}
& d_{r r}=e_{r r}+p_{r r}=u_{r, r} ; d_{\varphi \varphi}=e_{\varphi \varphi}+p_{\varphi \varphi}=\frac{u_{r}}{r} \\
& d_{z z}=e_{z z}+p_{z z}=0 ; \\
& e_{r \varphi}=p_{r \varphi}=0 ; e_{r z}=p_{r z}=0 ; e_{\varphi z}=p_{\varphi z}=0 .
\end{aligned}
$$

Используя данные соотношения, закон Гука $\sigma_{i j}=\lambda e_{k k} \delta_{i j}+2 \mu e_{i j}$ и уравнения равновесия $\sigma_{r r, r}+r^{-1}\left(\sigma_{r r}-\sigma_{\varphi \varphi}\right)=-\rho r \omega^{2}$, получим дифференциальное уравнение второго порядка для $u_{r}$ :

$$
u_{r, r r}+r^{-1} u_{r, r}-r^{-2} u_{r}=2 r^{-1} \frac{\mu}{\lambda+2 \mu}\left(p_{r r}-p_{\varphi \varphi}\right)+p_{r r, r}+\frac{\lambda}{\lambda+2 \mu}\left(p_{\varphi \varphi, r}+p_{z z, r}\right)-\frac{\rho \omega^{2}}{\lambda+2 \mu} r .
$$

В теории упругости уравнение представляет собой неоднородные уравнения Коши - Эйлера, и его общее решение имеет вид:

$$
\begin{aligned}
& u_{r}=c_{1} r+\frac{c_{2}}{r}-\frac{1}{8} \frac{\rho \omega^{2}}{\lambda+2 \mu} r^{3}, \quad \text { (3) } \begin{array}{c}
1) \text { с жестким включением и своб } \\
\text { границей }
\end{array} \\
& \left.u_{r}\right|_{r=R_{1}}=0 ;\left.\sigma_{r r}\right|_{r=R_{2}}=0 ; \\
& c_{1}=\frac{1}{8} \frac{\rho \omega^{2}}{\lambda+2 \mu} \frac{\mu R_{1}^{4}+(2 \lambda+3 \mu) R_{2}^{4}}{(\lambda+\mu) R_{2}^{2}+\mu R_{1}^{2}} ; c_{2}=-\frac{1}{8} \frac{\rho \omega^{2}}{\lambda+2 \mu} \frac{(2 \lambda+3 \mu) R_{2}^{2}-(\lambda+\mu) R_{1}^{2}}{(\lambda+\mu) R_{2}^{2}+\mu R_{1}^{2}} R_{1}^{2} R_{2}^{2} .
\end{aligned}
$$

где $c_{1}$ и $c_{2}-$ константы интегрирования.

Решим четыре краевые задачи, в которых коэффициенты интегрирования $c_{1}$ и $c_{2}$ примут следующий вид:

1) с жестким включением и свободной внешней

2) с жестким включением и жестко зафиксированной внешней границей

$$
\begin{aligned}
& \left.u_{r}\right|_{r=R_{1}}=0 ;\left.u_{r}\right|_{r=R_{2}}=0 ; \\
& c_{1}=\frac{1}{8} \frac{\rho \omega^{2}}{\lambda+2 \mu}\left(R_{2}^{2}+R_{1}^{2}\right) ; c_{2}=-\frac{1}{8} \frac{\rho \omega^{2}}{\lambda+2 \mu} R_{1}^{2} R_{2}^{2} .
\end{aligned}
$$

3) с внутренней полостью и жестко зафиксированной внешней границей

$$
\begin{aligned}
& \left.\sigma_{r r}\right|_{r=R_{1}}=0 ;\left.u_{r}\right|_{r=R_{2}}=0 ; \\
& c_{1}=\frac{1}{8} \frac{\rho \omega^{2}}{\lambda+2 \mu} \frac{\mu R_{2}^{4}+(2 \lambda+3 \mu) R_{1}^{4}}{(\lambda+\mu) R_{1}^{2}+\mu R_{2}^{2}} ; c_{2}=\frac{1}{8} \frac{\rho \omega^{2}}{\lambda+2 \mu} \frac{(\lambda+\mu) R_{2}^{2}-(2 \lambda+3 \mu) R_{1}^{2}}{(\lambda+\mu) R_{1}^{2}+\mu R_{2}^{2}} R_{1}^{2} R_{2}^{2} .
\end{aligned}
$$

4) с внутренней полостью и свободной внешней границей

$$
\begin{aligned}
& \left.\sigma_{r r}\right|_{r=R_{1}}=0 ;\left.\sigma_{r r}\right|_{r=R_{2}}=0 ; \\
& c_{1}=\frac{1}{8} \frac{\rho \omega^{2}}{\lambda+2 \mu} \frac{2 \lambda+3 \mu}{\lambda+\mu}\left(R_{2}^{2}+R_{1}^{2}\right) ; c_{2}=\frac{1}{8} \frac{\rho \omega^{2}}{\lambda+2 \mu} \frac{2 \lambda+3 \mu}{\mu} R_{1}^{2} R_{2}^{2} .
\end{aligned}
$$

Рассмотрим деформацию ползучести. Для ее определения воспользуемся степенным законом Нортона:

$$
\dot{p}_{i j}=\varepsilon_{i j}^{v}=\frac{\partial V}{\partial \sigma_{i j}} ; V\left(\sigma_{i j}\right)=B \Sigma^{n}\left(\sigma_{1}, \sigma_{2}, \sigma_{3}\right) ; \Sigma=\frac{1}{\sqrt{2}} \sqrt{\left(\sigma_{r r}-\sigma_{\varphi \varphi}\right)^{2}+\left(\sigma_{\varphi \varphi}-\sigma_{z z}\right)^{2}+\left(\sigma_{z z}-\sigma_{r r}\right)^{2}},
$$

где $V$ - потенциал ползучести, $B, n-$ параметры материала, $\Sigma-$ интенсивность напряжений.

Выполнив преобразования, получим:

$$
\dot{p}_{i j}=\varepsilon_{i j}^{v}=\frac{3}{2} B n \Sigma^{n-2} \tau_{i j},
$$


Примем, что в случае начала пластического течения скорости необратимых деформаций $\dot{p}_{i j}$ будут складываться как из скоростей деформации ползу- чести $\varepsilon_{i j}^{v}$, так и пластичности $\varepsilon_{i j}^{p}$. В качестве модели пластического течения возьмем модель вязко-пластичности

$$
\varepsilon_{i j}^{p}=\xi \frac{\partial f}{\partial \sigma_{i i}} ; f\left(\sigma_{i j}, \varepsilon_{i j}^{p}\right)=\Sigma-2(k+\eta \mathrm{E})=0 ; \mathrm{E}=\frac{1}{\sqrt{2}} \sqrt{\left(\varepsilon_{r r}^{p}-\varepsilon_{\varphi \varphi}^{p}\right)^{2}+\left(\varepsilon_{\varphi \varphi}^{p}-\varepsilon_{z z}^{p}\right)^{2}+\left(\varepsilon_{z z}^{p}-\varepsilon_{r r}^{p}\right)^{2}},
$$

где $\varepsilon_{i j}^{p}$ - компоненты тензора скоростей пластической деформации, $\xi-$ положительный множитель, $f$ - пластический потенциал, А - интенсивность скорости пластических деформаций, $\eta$ - коэффициент вязкости.

Преобразовав уравнения и найдя значение коэффициента, в итоге получим соотношения для нахождения скорости пластической деформации

$$
\varepsilon_{i j}^{p}=\frac{1}{2 \eta} \frac{\Sigma-2 \mathrm{k}}{\Sigma} \tau_{i j}
$$

\section{Результаты расчетов}

Решалась задача разгона цилиндрической среды по закону $\omega=\omega_{\max } \frac{1}{2}\left(1-\cos \left(\frac{\pi t}{t_{1}}\right)\right)$, где $t_{1}$ - время разгона, $\omega_{\max }-$ максимальная скорость вращения. Рассматривалось решение задачи разгона за один час

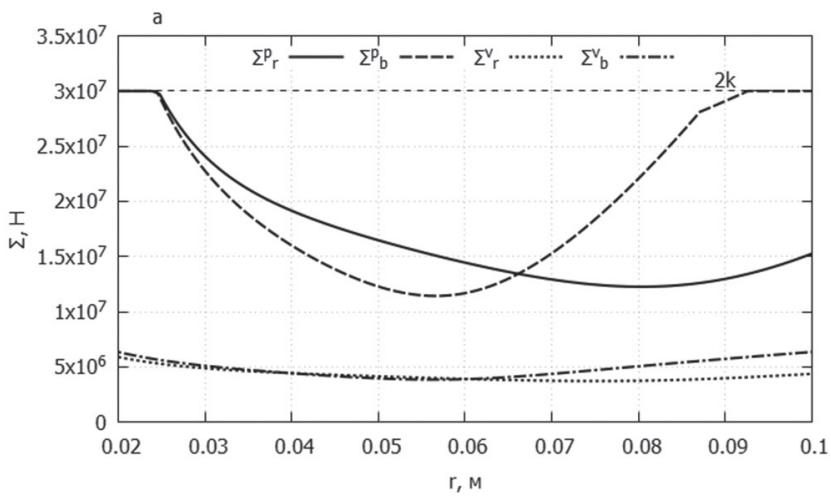

Рис. 1. Распределение интенсивностей напряжений $u_{r}$ в средах: $\mathrm{a}-\mathrm{c}$ жесткой левой границей; $6-$ со свободной левой границей
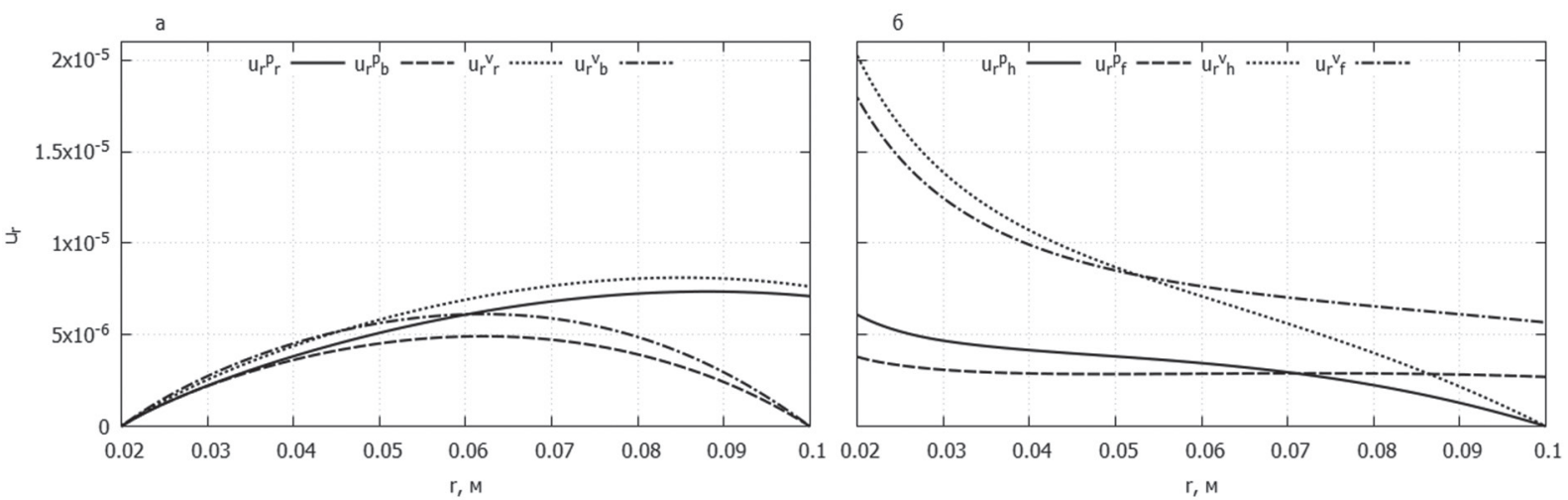

Рис. 2. Распределение перемещений $u_{r}$ в средах:

$\mathrm{a}-\mathrm{c}$ жесткой левой границей; $6-$ со свободной левой границей

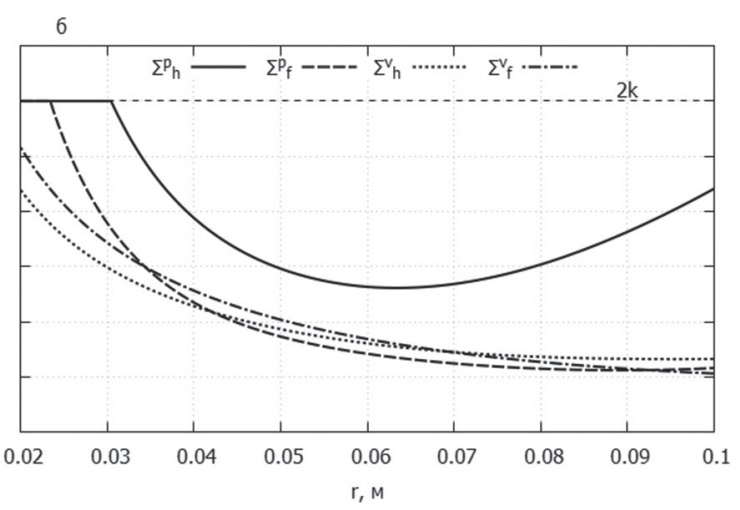

при ползучести и, для сравнения с полученными результатами, упруго вязкопластическое решение задачи разгона среды. Также для достижения примерно равных значений интенсивности напряжений были взяты различные максимальные значения скорости вращения для разных граничных условий.

В результате при ползучести наблюдаем снижение напряжений. В особенности этот эффект проявляется в задачах с зафиксированной внутренней границей (рис. 1, a). В меньшей степени он наблюдается в задаче полого цилиндра с зафиксированной внешней границей. В случае свободного цилиндра наблюдается перераспределение напряжений по объему (рис. 1, б). Изменения в перемещениях, наоборот, в первом случае малы (рис. 2, а), а во втором - велики (рис. 2, б). При этом максимальные значения исходных перемещений $u_{r}^{p}$ примерно равны. 
Для оценки необратимых деформаций воспользуемся инвариантом тензора необратимых деформаций: $P_{2}=\sqrt{p_{r r}^{2}+p_{\varphi \varphi}^{2}+p_{z z}^{2}-p_{r r} p_{\varphi \varphi}-p_{\varphi \varphi} p_{z z}-p_{r r} p_{z z}}$. Как можно видеть из графиков, для сред с жестким включением

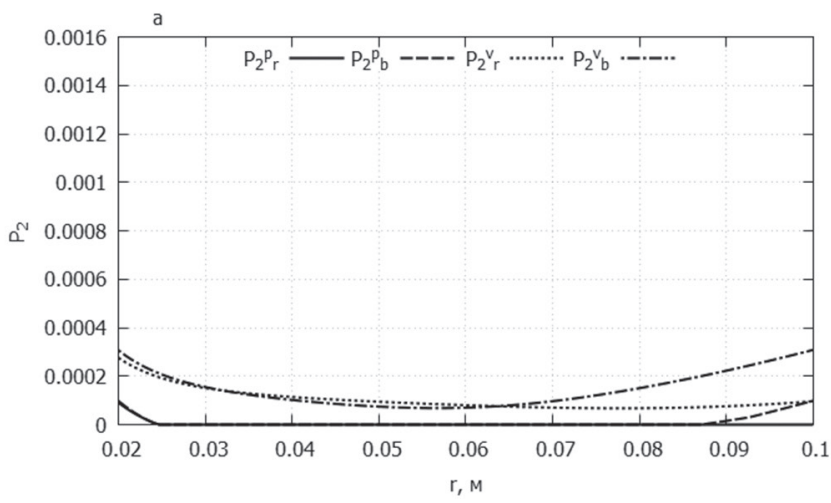

необратимые деформации растут на порядок медленней (рис. 3, а), чем для сред с внутренней полостью (рис. 3,6$)$. Также они более равномерно распределены по всей среде с максимальным значением у зафиксированных границ. Для сред с внутренней полостью необратимые деформации сосредоточены у левой границы.

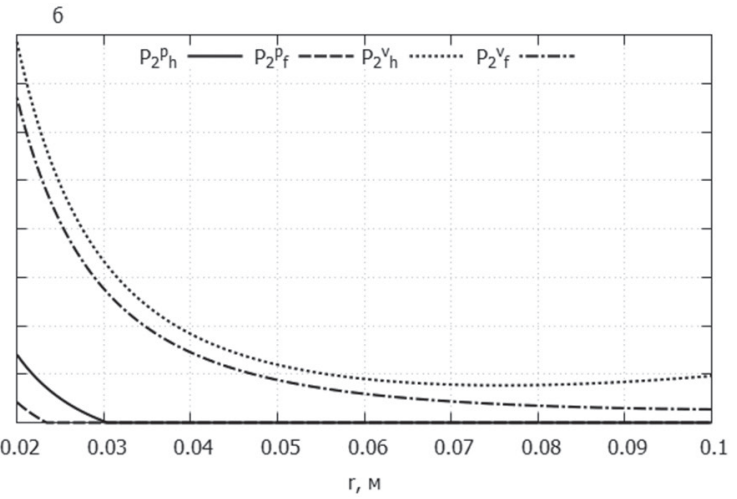

Рис. 3. Значение инварианта $P_{2}$ в средах:

$\mathrm{a}-\mathrm{c}$ жесткой левой границей; $6-$ со свободной левой границей

\section{Заключение}

При долгом разгоне происходит снижение интенсивности напряжений в связи с накоплением необратимых деформаций. Особо сильно это заметно в цилиндрических средах с жестко закрепленной левой границей. В свободной среде вместо снижения интенсивности напряжения происходит ее перераспределение и накопление перемещений. Также необратимые деформации в средах с жестким включени- ем растут более равномерно с трехкратной разницей между максимальными и минимальными значениями. В средах с полостью наблюдается значительный рост максимума и примерно равный - минимума, по сравнению с первыми средами. Следует отметить, что во всех задачах наблюдается снижение максимального значения интенсивности напряжений, что позволяет избежать появления области пластического течения.

\section{Библиографический список}

1. Nadai A. Theory of Flow and Fracture of Solids, Volume One. 2nd Edition. - McGraw Hill, 1950.

2. Gamer U., Mack W., Varga I. Rotating elastic-plastic solid shaft with fixed ends // International Journal of Engineering Science. - 1997. - Vol. 35. - № 3. DOI: 10.1016/S00207225(96)00085-7

3. Hodge P.G., Balaban M. Elastic-plastic analysis of a rotating cylinder // International Journal of Mechanical Sciences. - 1962. - Vol. 4. - № 6. DOI: 10.1016/S00207403(62)80008-3

4. Работнов Ю.Н. Ползучесть элементов конструкций. - М., 1966.

5. Bhatnagar N.S., Kulkarni P.S., Arya V.K. Creep analysis of an internally pressurised orthotropic rotating cylinder// Nuclear Engineering and Design. - 1984. - Vol. 83. - № 3. DOI: 10.1016/0029-5493(84)90130-4

6. Bhatnagar N.S., Arya V.K., Debnath K.K. Creep An alysis of Orthotropic Rotating Cylinder // J. Pressure Vessel Technol. — 1980. — Vol. 102. — № 4. DOI: 10.1115/1.3263347
7. Bhatnagar N.S., Kulkarni P.S., Arya V.K. Creep analysis of orthotropic rotating cylinders considering finite strains // International Journal of Non-Linear Mechanics. - 1986. Vol. 21. - № 1. DOI: 10.1016/0020-7462(86)90013-2

8. Bose T., Rattan M. Effect of thermal gradation on steady state creep of functionally graded rotating disc // European Journal of Mechanics - A/Solids. - 2018. - Vol. 67. № Supplement. DOI: 10.5281/zenodo.1131585

9. Mangal S.K., Kapoor N., Singh T. Steady-State Creep Analysis of Functionally Graded Rotating Cylinder // Strain. 2013. - Vol. 49. — № 6. DOI: 10.1111/str.12052

10. Бажин А.А., Буренин А.А., Мурашкин Е.В. К моделированию процесса накопления больших необратимых деформаций в условиях пластического течения и ползучести // Прикладная математика и механика. - 2016. - Т. 80. — № 2.

11. Бегун А.С., Буренин А.А., Ковтанюк Л.В., Панченко Г.Л. Развитие и торможение вязкопластического течения с учетом ползучести материалов упругих зон // Вестник ДВО РАН. — 2016. — № 4. 